\title{
Radio-frequency plasma polymerized biodegradable carrier for in vivo release of cis-platinum
}

\author{
Sudhir Bhatt ${ }^{1,3}$, Fatemeh Valamanesh ${ }^{2}$, Jerome Pulpytel ${ }^{1}$, Rea Lo Dico ${ }^{2}$, Aliby \\ Baiyukha $^{1}$, Iman Al-dybiat ${ }^{2}$, Marc Pocard ${ }^{2}$, Farzaneh Arefi-Khonsari ${ }^{1}$, Massoud \\ Mirshahi $^{2}$ \\ ${ }^{1}$ Sorbonne Universités, UPMC Univ. Paris 6, CNRS, Laboratoire Interfaces et Systèmes Electrochimiques, 75005, Paris, \\ France \\ ${ }^{2}$ Sorbonne Paris Cité Universités, UMR Univ. Paris 7, INSERM U965 Carcinose, Angiogenèse et Recherche Translationnelle, \\ L'Hôpital Lariboisière, 75010, Paris, France \\ ${ }^{3}$ Current address: Frank Reidy Research Center for Bioelectrics, Old Dominion University, Norfolk, 23508, VA, USA \\ Correspondence to: Massoud Mirshahi, email: massoud.mirshahi@inserm.fr \\ Keywords: plasma polymers, biodegradable coatings, in vivo drug delivery system, anti-cancer drug, targeted therapy \\ Received: May 11,2016_Accepted: July 19, $2016 \quad$ Published: July 29, 2016
}

\section{ABSTRACT}

A low pressure plasma process based on plasma deposition has been used to develop a drug delivery strategy. In this study, a drug delivery system based on different layers of plasma co-polymerized Poly $\varepsilon$-caprolactone-Polyethylene glycol (PCL-PEG) co-polymers was deposited on biocompatible substrates. Cis-platinum $\left(118 \mathrm{\mu gm} / \mathrm{cm}^{2}\right)$ was used as an anti-cancer drug and incorporated for local delivery of the chemotherapeutic agent. The co-polymer layers and their interaction with cancer cells were analyzed by scanning electron microscopy. Our study showed that the plasma-PCL-PEG coated cellophane membranes, in which the drug, was included did not modify the flexibility and appearance of the membranes. This system was actively investigated as an alternative method of controlling localized delivery of drug in vivo. The loading of the anti-cancer drug was investigated by UV-VIS spectroscopy and its release from plasma deposited implants against BALB/c mice liver tissues were analyzed through histological examination and apoptosis by TUNEL assay. The histological examination of liver tissues revealed that when the plasma-modified membranes encapsulated the cis-platinum, the Glisson's capsule and liver parenchyma were damaged. In all cases, inflammatory tissues and fibrosis cells were observed in contact zones between the implant and the liver parenchyma. In conclusion, low pressure plasma deposited uniform nano-layers of the co-polymers can be used for controlled release of the drug in vivo.

\section{INTRODUCTION}

In the past several years interest in platinum drugs has increased due to their successful administration for the treatment of most disseminated cancers [1]. Cisplatinum (cis-diaminedichloroplatinum (II)) remains one of the most widely used and potent anticancer agents against many different types of cancer [2-3]. Cis-platinum exerts its anticancer effects by covalently binding to DNA and forming various platinum-DNA adducts which cause distortions in DNA and that leads to final cellular outcome of apoptotic cell death [4]. For the cancer treatment, cis-platinum is usually intravenously administered but it is often unsuccessful because of the nonselective distribution of the drug among the normal and tumor tissue and that likely enhances the risk of dose limiting side effects including chronic neurotoxicity, acute nephrotoxicity and myelosuppression [5-6]. After $24 \mathrm{hrs}$ of clinical administration of cis-platinum, it has been noticed that more than $65 \%$ of the platinum in the blood was protein bound, leading to severe side effects of cis-platinum treatment and therefore less therapeutic efficacy [7] and resistance over the course of medication [8]. In order to overcome these therapeutic limitations by 
cis-platinum treatment and other anticancer drugs, there is a clear incentive to develop new strategies for efficient, systematic and controlled release of cis-platinum for oncology.

Towards enhancement for the prevention of the systematic side effects of the chemotherapy, a wide range of strategies have been developed for a more selective delivery. The trend in drug delivery technology has been toward biodegradable polymer excipients requiring no follow-up surgical removal once the drug supply is depleted [9]. For instance, wet chemically prepared polymer based cis-platinum loaded drug delivery systems such as platinum encapsulated PLGA-b-PEG nanoparticles [10], liposomes [11], PEG-b-poly (amino acid)-based polymeric micelles [12], polymeric conjugates of $\gamma$-PGA-cis-platinum [13], $\mathrm{pH}$ sensitive Bi(PEG-PLA)$\mathrm{Pt}(\mathrm{IV})$ polymer-prodrug conjugates [14] etc. The recent advances in nanoparticle formulations of cis-platinum for therapeutic applications have been thoroughly surveyed and the clinical utility of cis-platinum conjugates have been investigated [15].

As compared to the wet processes [9-16], plasma polymerization is a catalyst and solvent free, dry, one step, highly controllable and environmentally benign process. Recently, the radio frequency (RF) plasma (co-) polymerization of different organic precursors for surface modifications of a variety of substrates have been summarized in order to tailor the physico-chemical properties of the substrates for mitigation of nonspecific adsorption of proteins, tunable biomolecule-surface interactions and controlled drug delivery applications $[17,18]$. The synergetic role of plasma generated free radicals for tuning the surface properties of polymers and their applications have been discussed broadly for drug delivery, biomedical and tissue engineering [19].

The biodegradable and biocompatible PCL-co-PEG polymers with different cell adhesion and cell-repellent properties have been prepared on different flat substrates by gradually varying the partial pressure ratio of the monomers ( $\varepsilon$-CL/DEGME) under the solvent and catalyst free low-pressure inductively excited RF plasma reactor [20-23]. Later, cis-platinum loaded multilayer of plasma was studied polymerized PCL-co-PEG coatings have been used to produce barrier layers on different flat surfaces (glass, Si wafer, etc.) for the controlled release of drug and the in vitro release of anticancer drug was investigated for the apoptosis of ovarian cancer cells [22].

In our previous works [20-23], we have reported how to develop a Drug Delivery System based on multiple layers composed of plasma copolymers deposited on glass, and $\mathrm{Si}$ wafer by the dry clean plasma process. The controlled drug delivery of anticancer drug was investigated in-vitro for the apoptosis of ovarian cancer cells [22]. Here the same drug delivery system was deposited on cellophane membranes and the controlled release of cis-platinum was studied in vivo.

\section{RESULTS}

\section{UV-visible spectroscopic measurements for cis- platinum loading}

The loading of cis-platinum on plasma copolymerized cellophane substrates was determined by measuring the absorbance in the range of $200-500 \mathrm{~nm}$ using UV-Vis spectrometer. The absorption spectrum of $1 \mathrm{mM}$ cis-platinum in $1 \mathrm{X}$ PBS, $\mathrm{pH} 7$, is shown in Figure 1. Because of metal to ligand charge transfer (MLCT) transitions in cis-platinum solution [23], a strong band at $203 \mathrm{~nm}$ and a shoulder at $229 \mathrm{~nm}$ are appeared. Cis-platinum compound exhibits a much weaker band at $330 \mathrm{~nm}$ which is assigned to the $\mathrm{d}-\mathrm{d}$ transition of $\mathrm{Pt}^{2+}$ ions.

\section{Cell-membrane contact analysis by scanning electron microscopy}

As presented in Figure 2A, uniform copolymer layer (UCL) is intact and posed correctly on the substrate. Ovarian cancer cell line OVCAR-3 adhered on the UCL in the cell culture condition and do not perturbed by culture medium. Adhered cells form the filopodias on the membrane (Figure 2B). Incorporation of cis-platinum strongly modified the physiological properties of UCL by induction of cells detachment (Figure 2C). As presented in Figure 2D, the cellular debris remained after cell death on the UCL.

\section{Influence of multilayer coatings membrane implants on the liver parenchyma}

In our previously published work [22], the cisplatinum loaded plasma polymerized multilayer PCLco-PEG coatings was assessed in vitro using human ovarian carcinoma cells (NIH: OVCAR-3) where it has been demonstrated that controlled release of cis-platinum was effectively induced apoptosis. Herein, after 10 days of hepatic implantation, the mice liver was extracted by surgical resection and histological measurements were performed (Figure 3). In Figure 3A, 1-3, the macroscopic and representative image of cellophane implanted animal, the position of the implant on the liver, magnified image showing surgically removed implant and representative image of histology are presented. Figure 3B, 1-4, histological examination of plasma deposited implants wrapped onto a liver, showed that the implants were properly positioned and surrounded by a fibrous capsule. This capsule was homogeneous along the whole perimeter of the plasma coated implants, with limited areas of necrosis and inflammatory cells. Several liver sections from this region were analysed for each case (Figure 3B, 1-4). Normal (3B-1) and the liver exposed to bare cellophane, unloaded drug membranes were presented in Figure 3B, 1-3. The liver is invested 


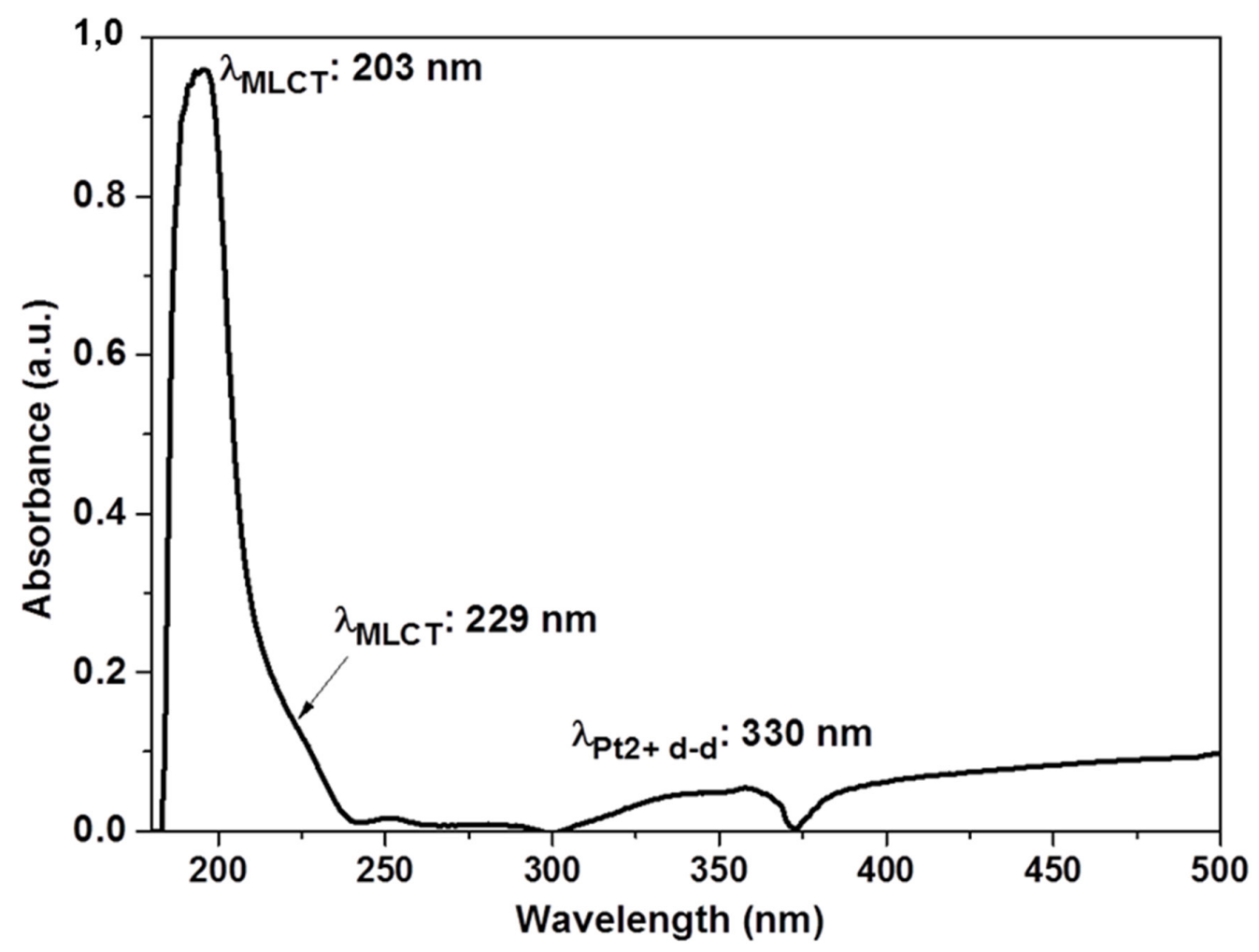

Figure 1: UV absorption spectrum of $1 \mathrm{mM}$ Cis-platinum in $1 \mathrm{X}$ PBS at pH 7.
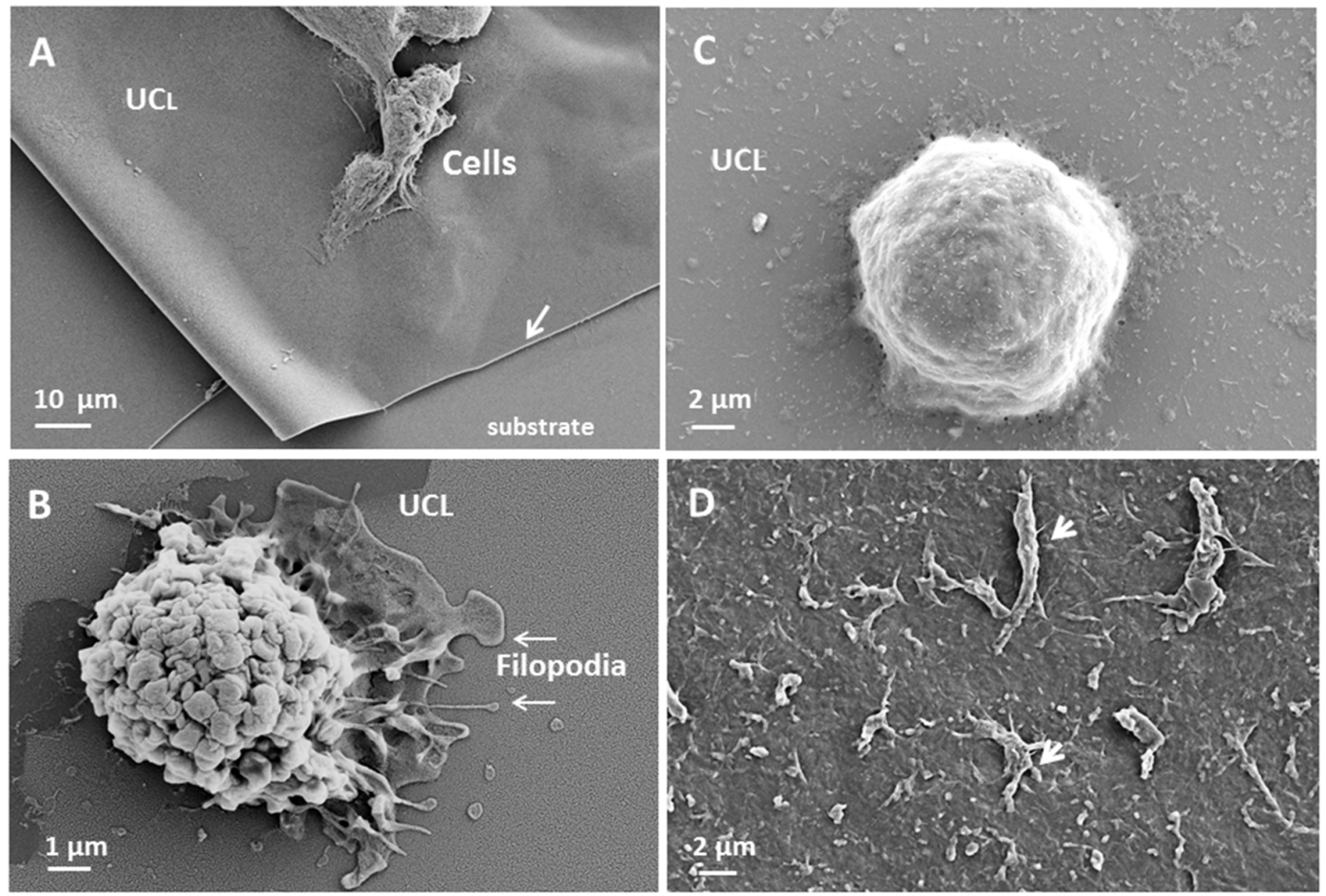

Figure 2: interaction of ovarian cancer cell with uniform copolymer layer (UCL). Ovarian cancer cell line OVCAR-3 adhered on the UCL (arrow) in the culture medium (A. X2500). This interaction is biocompatible and adhered cell form the pseudopod and nanopod on the UCL (B. X20000). When the anti-cancer drug was incorporated, on the UCL, the cells shape changed (C. x10000) and adherent necro-apoptotic cell organelles (arrow) were observed after cells detachments (D. X5000). 
A

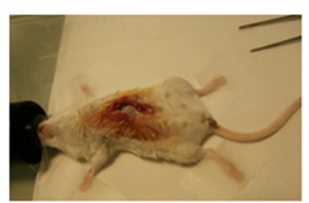

2

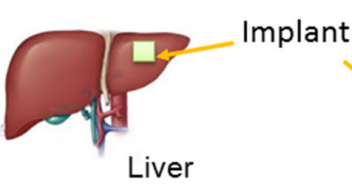

3

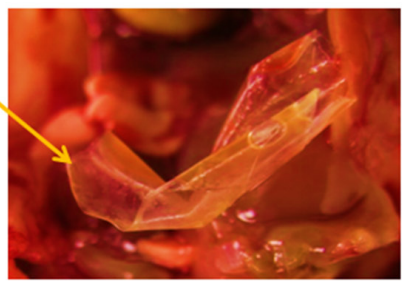

4

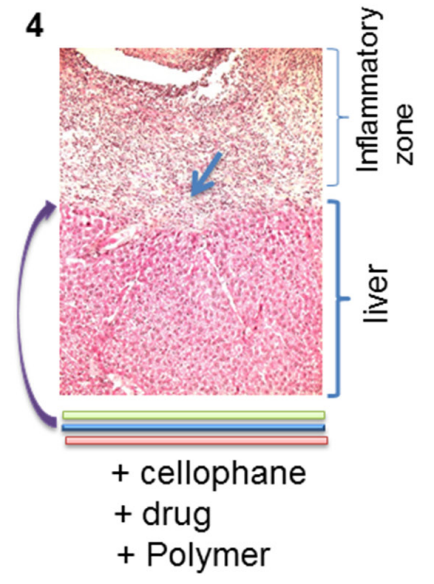

C

2

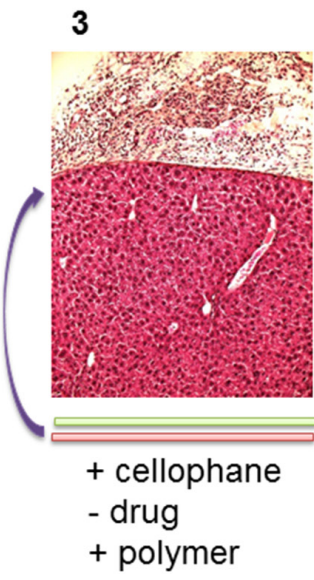

4

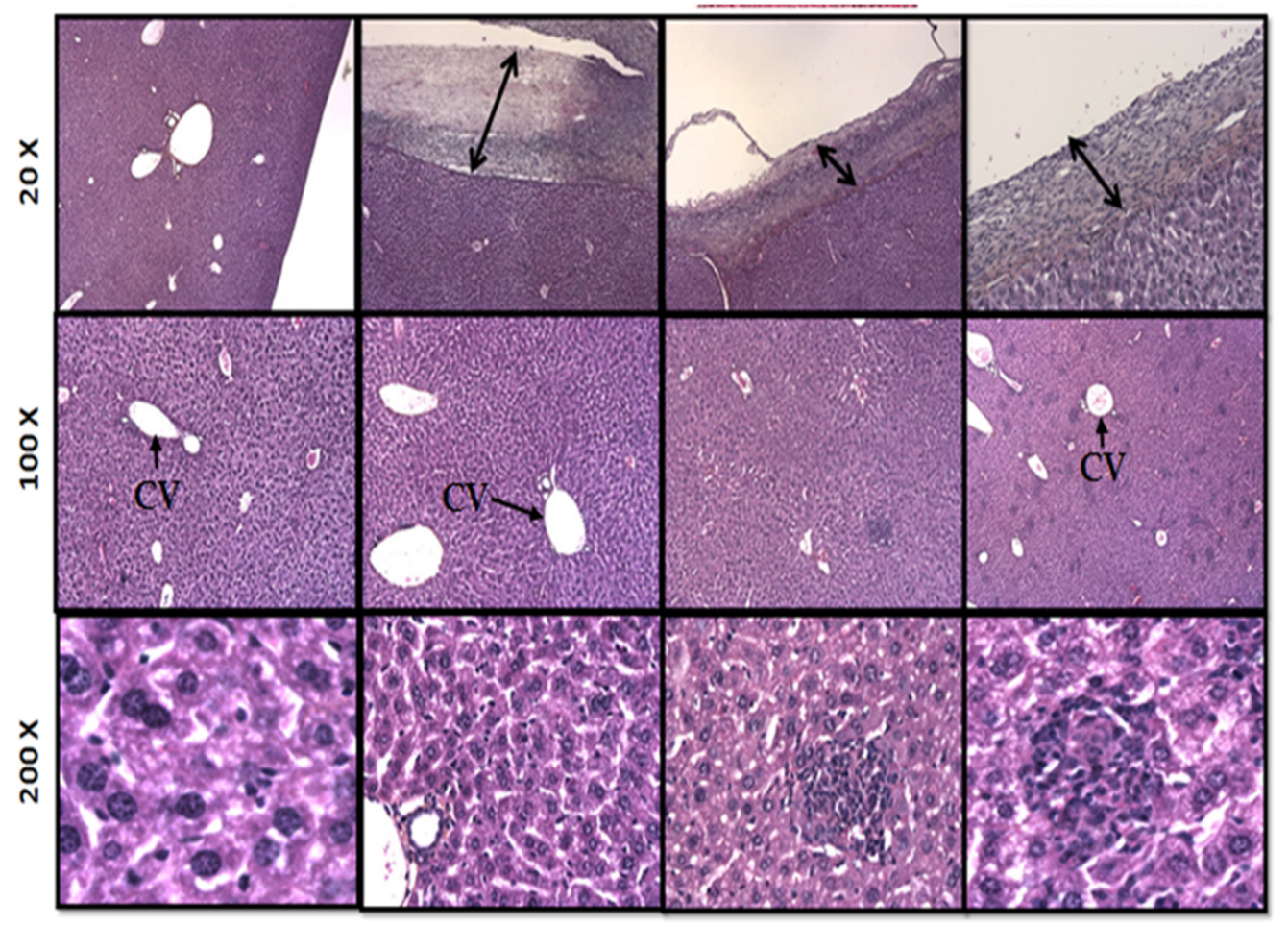

Figure 3: Macroscopic and microscopic observation of hepatic implantation of cellophane inBALB/c mice sacrificed on the $10^{\text {th }}$ day. Macroscopic and representative image of cellophane implanted animal, magnified image showing surgically removed implant and representative image of histology A. Microscopic images for the histological analysis of normal liver tissues called of control B-1. liver exposed to untreated (uncoated) cellophane (Cello) B-2. plasma polymers + cellophane coated with copolymer without cisplatinum (4B-3) cellophane ( $p P C L-c o-P E G$-Cello) B-3. cellophane coated with copolymer + drug and plasma polymers, + Cis-platinum + cellophane (Cisp-pPCL-co-PEG-Cello) B-4. Data are a typical microscopic imaging of liver tissue sections C. under original magnification of $20 \mathrm{X}, 100 \mathrm{X}$ and $200 \mathrm{X}$. Nuclei: dark blue, cytoplasm: pink. CV: Central vein. 
by a connective tissue capsule named Glisson's capsule. The histological examination of the liver of the control mice exhibited the normal architecture of the liver whereas the parenchyma of the control liver specimens was uniform in appearance. As presented in Figures 3B2, when untreated cellophane was used, the Glisson's capsule and liver parenchyma were intact after 10 days. In case of $p P C L$-co-PEG-cello, no significant change was observed in liver tissues as compared to control Figures 3B-3. That shows the plasma polymerized PCLco-PEG coatings on cellophane implants which were in contact with liver for 10 days, were biocompatible and did not generate significant inflammatory response. The histological appearance of parenchyma in $\mathrm{pPCL}-\mathrm{co}$ $P E G$-Cello, and untreated cellophane was comparable with that of control group. On the contrary, when the membrane contained cis-platinum (Cisp-pPCL-co-PEGCello,), the Glisson's capsule and liver parenchyma were damaged (Figure 3B-4). In addition, the mice liver treated with the Cisp-pPCL-co-PEG-Cello, formulation did exhibit inflammatory cells as compared to the control. As compared to control, the histological examination of the liver tissues of the Cisp-pPCL-co$P E G$-Cello treated mice exhibited congestion of the central veins and intracytoplasmic vacuolations were observed which might be due to the cis-platinum induced oxidative damage and lipid peroxidation of the liver tissues [24]. In the cells around the central vein (arrow $\mathrm{CV}$ ), the cytoplasmic modifications were observed. As shown in Figure 3C (magnification: 200X), the clusters of inflammatory cells were observed in the surrounding of portal region. In all implanted cases, the fibrosis was observed on the zones in contact with the implant and liver parenchyma showed by arrow (Figure 3C, 2-4).

\section{Detection of apoptotic cells in liver tissues}

In order to detect the apoptosis in the liver tissues, the TUNEL immunohistological assay was performed for different experimental groups (Figure 4). As shown in stained sections (Figure 4A and 4B), no significant morphological differences were observed in liver tissues from mice implanted with untreated cellophane (Figure 4A) and plasma treated cellophane (Figure 4B). No apoptotic cells were observed with untreated cellophane (Cello) and pPCL-co-PEG-Cello. TUNEL positive (green florescence) signals were, however observed in liver tissues of mice wrapped with Cisp-pPCL-co-PEG-Cello implants for 10 days (Figure 4C). The magnified image of TUNEL positive cells in liver tissues is shown in Figure 4D.

\section{Cis-platinum traces in blood plasma by ultraviolet-visible spectrophotometry}

Preliminary studies were carried out to determine the traces of cis-platinum in the blood plasma in the broad ultraviolet-visible (UV-Vis) region ranging from 200 to $500 \mathrm{~nm}$. Initial studies were conducted using $0.2-$ $0.5 \mathrm{ml}$ of blood plasma from experimental groups and control group in a quartz cuvette. As presented in Figure 5 , The UV-V of the spectrum of the blood plasma shows a strong band at $410 \mathrm{~nm}$ and a less intense band at 250 $\mathrm{nm}$ attributed to the hemoglobin $(\mathrm{Hb})$ and oxidized $\mathrm{Hb}$ respectively. In the case of Cisp-pPCL-co-PEG-Cello, a very weak peak corresponding to $\mathrm{Pt}^{+2}$ ion can be identified at $330 \mathrm{~nm}$, even though it is not completely distinguishable from the rest of the spectra due to the overlapping. Therefore it is difficult to assign the absorption of platinum compounds in biological medium such as blood. One can note in Figure 2 which shows the UV absorption of cis-platinum in PBS, (Figure 5). It can be pointed out that the absorption spectrum of cisplatinum in blood plasma (Figure 5) is quite different as compared to its absorption in PBS (Figure 2). Therefore it is difficult to assign absorption of platinum compounds in biological media such as blood.

\section{DISCUSSION}

In this study we described the in vivo local drug release efficacy of the low pressure plasma polymerized PCL-co-PEG encapsulated cis-platinum implants as biocompatible and biodegradable drug carriers. A drug delivery system based on different layers presenting different chemical compositions of PCL-PEC copolymers obtained by low pressure plasma has been already reported [22]. The total thickness of these layers is in the order of a few hundreds of nm. However the first layers deposited to increase the affinity of the membrane is a few tens of nm, followed by a dense barrier deposited at higher powers, covered again by coatings under conditions which would be cell nonadhesive (Figure 6B). Indeed the reason why we have used PCL-PEG(1:4) is because the copolymers deposited under such conditions when deposited at low power can be cell nonadhesive while at higher powers they are dense and crosslinked and act as a barrier for leaching out of the drugs. In this work such multi-layer coatings have been deposited on biocompatible cellophane substrates which were flat, non-porous surfaces (2D). Cis-platinum loaded (118 $\mu \mathrm{gm} / \mathrm{cm}^{2}$ ) PCL-co-PEG coatings with optimized ratios of $\varepsilon$-CL/DEGME monomer feed in the plasma reactor were prepared for controlled cell death applications. The plasma treatments did not modify the flexibility and appearance of the membranes. As demonstrated by SEM, the copolymer layer was identifiable and the cancer cells forming the pods adhered well on the copolymer. These results suggest the biocompatibility and non-cytotoxicity of the membrane complex in the absence of drug. After incorporation of the drug, the membrane becomes cytotoxic. Cis-platinum-loaded cellophane implants were placed directly at the site of 
mice liver, offering a controlled and local release of drug by means of polymer swelling and/ or dissolution and/or disintegration. The UV-Vis spectroscopic measurements were conducted on both cis-platinum solution in PBS (drug loading) and cis-platinum release in blood plasma. By using UV absorption measurements, we showed that cis-platinum reacted with proteins in the blood plasma and a derivatization technique is required for the quantification of cis-platinum in blood stream. By the different biochemical techniques, the reaction abilities of the platinum complexes such as cis-platinum to blood serum proteins have been reported before [25]. As compared to other proteins such as albumin and immunoglobulin, higher reaction (binding) ability of cis-platinum to hemoglobin $(\mathrm{Hb})$ has been shown [26]. In our case of cis-platinum in blood plasma, a high absorbance of $\mathrm{Hb}$ was obtained at $410 \mathrm{~nm}$ as compared to the intensity of the cis-platinum for $\mathrm{Pt}^{+2}$ at $330 \mathrm{~nm}$. After 10 days of implantation, the detection of traces of cis-platinum in the blood plasma might be difficult by means of UV absorption measurements without carrying out derivatization which result from the in vivo formation of cis-platinum-protein adducts via strong interactions between blood plasma and platinum ligands. Due to the complexity of the blood plasma and the low molecular absorptivity of cis-platinum in the UV region, the selective derivatization for the detection of cis-platinum in biological samples has been discussed by means of optical measurements of the platinum-ligand drugs [27].

Our previous in vitro study using plasma polymerized PCL-co-PEG coated cis-platinum for controlled release has confirmed the cytotoxic effects of cis-platinum to cancer cells [22].

Then the cellulose based polymer was covered with the multi-layered coating according to the procedure explained and was implanted on Glisson's capsule of the liver. The in vivo measurements showed that all animals reacted against the implant and after 10 days of post-implantation, inflammatory tissues were generated in the region of implants. Consequently, macroscopic and microscopic analysis demonstrated endogenous tissues containing the micro-vessels, fibrocytes, immune inflammatory cells, tissues matrix and fibrosis. In all experiments except when the implant
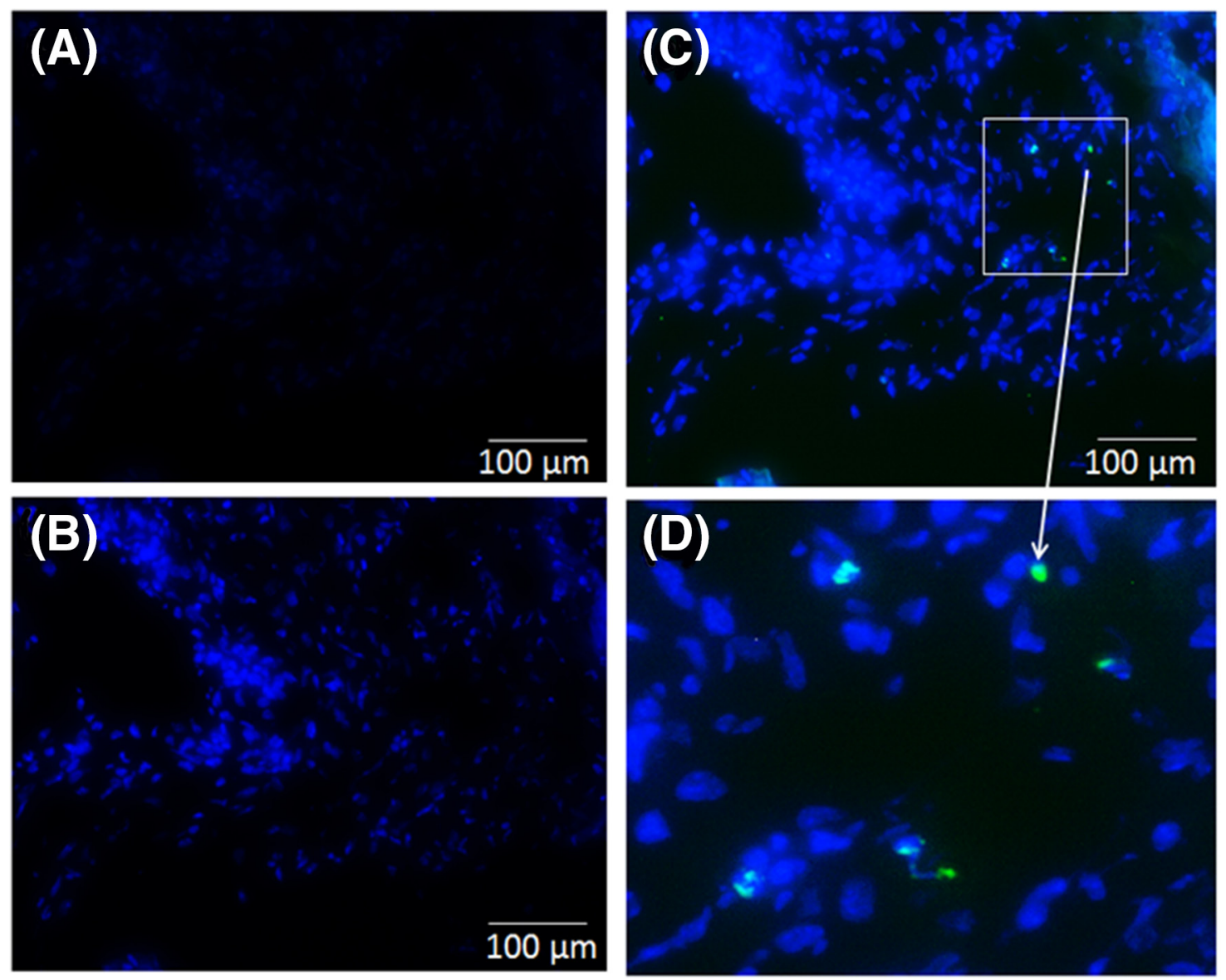

Figure 4: Apoptotic cells in liver tissue sections were detected using TUNEL assay for untreated cellophane (Cello) A. plasma polymers + cellophane $(p P C L-c o-P E G$-Cello $)$ B. plasma polymers + Cis-platinum + cellophane $(C i s p-p P C L-c o-P E G-$ Cello) C. and magnified image showing apoptotic cells D. Data are a typical fluorescent microscopic imaging of liver tissue sections under original magnification of $\times 100$. Nuclei: blue, apoptosis cells: green. 


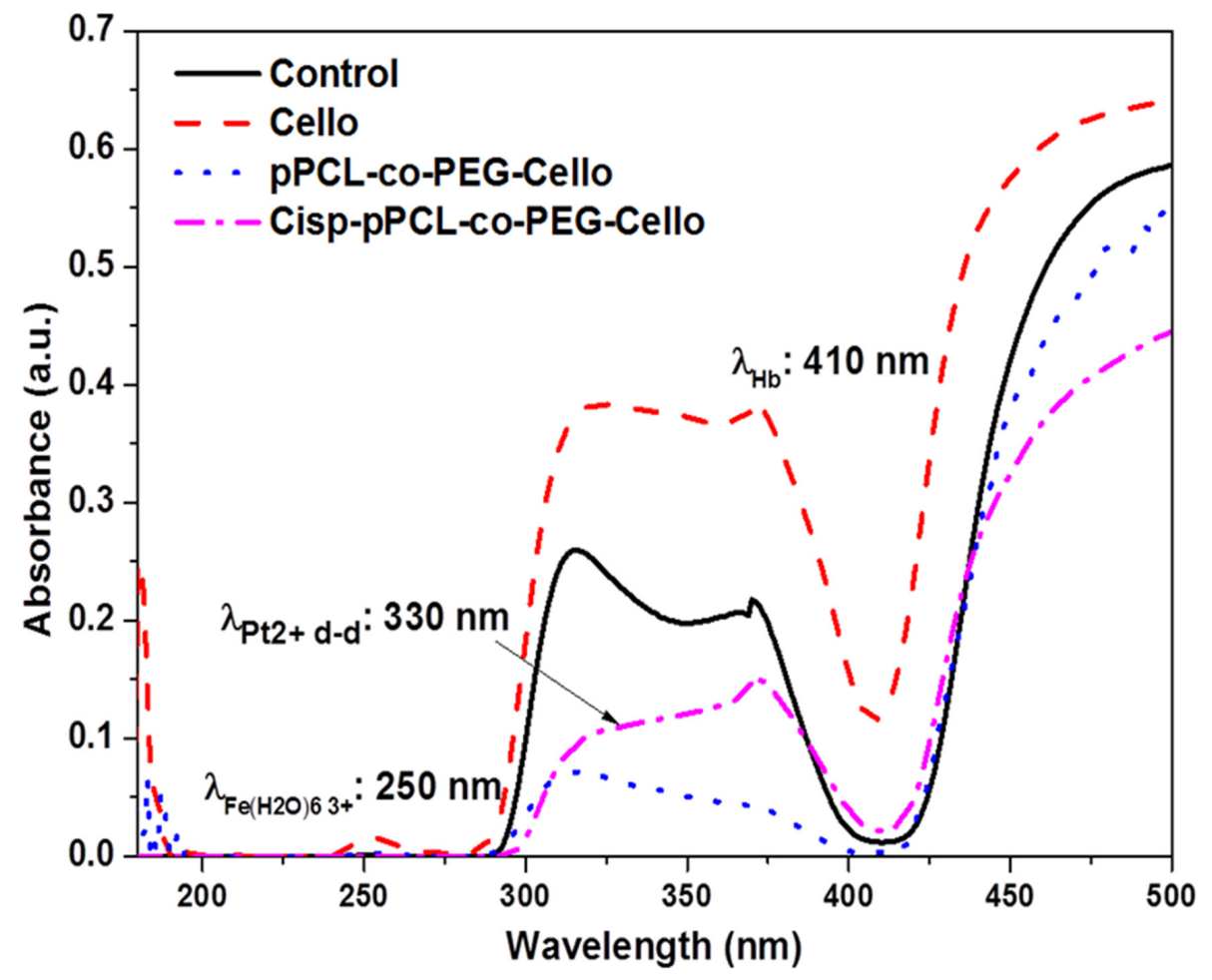

Figure 5: UV absorption spectrum of blood plasma for control, untreated cellophane (Cello), plasma polymers + cellophane (pPCL-co-PEG-Cello), and plasma polymers + Cis-platinum + cellophane (Cisp-pPCL-co-PEG-Cello).
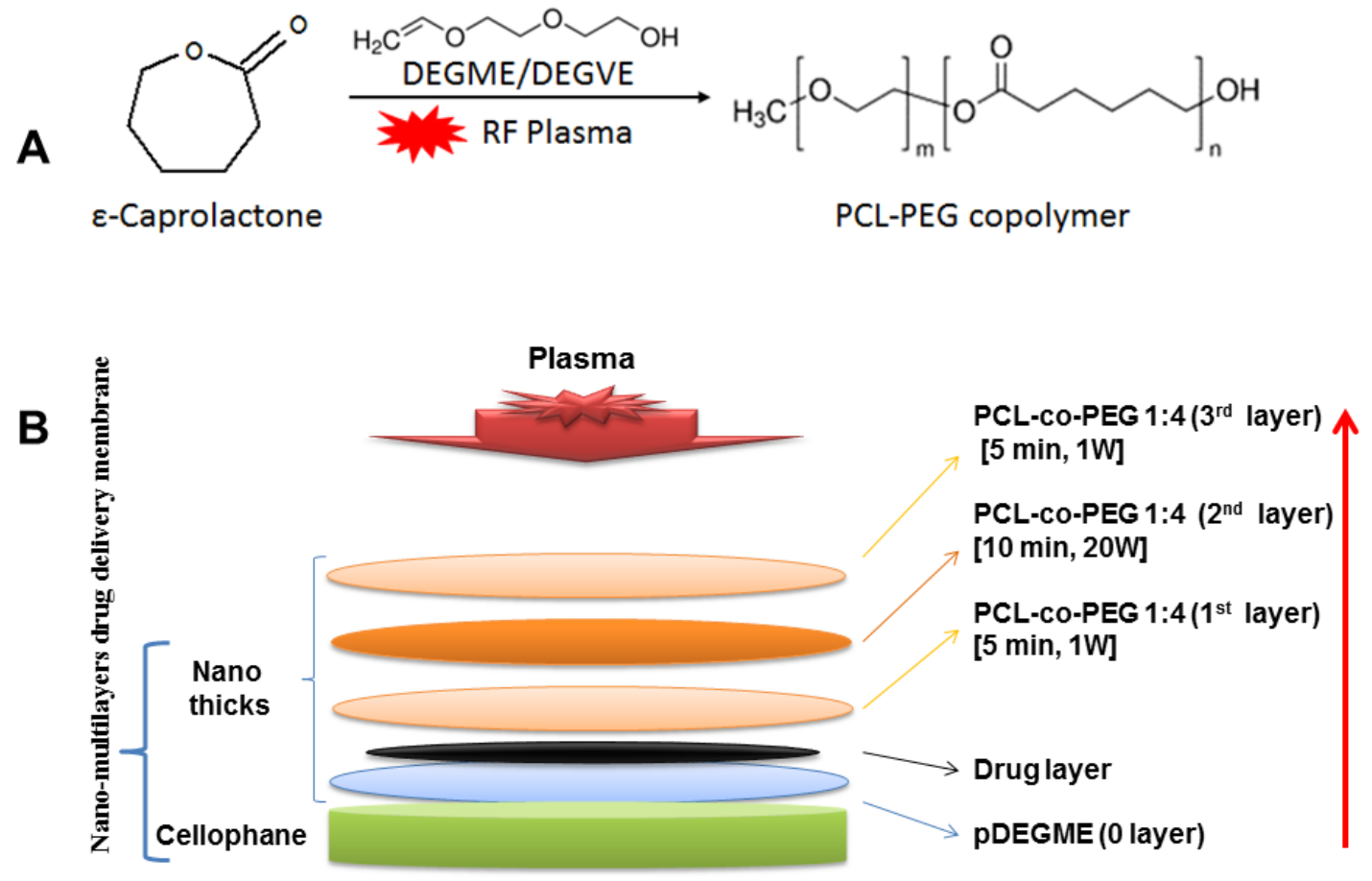

Figure 6: Schematic of plasma polymerized multilayer coatings on cellophane for in vivo release of cis-platinum. A. Radio-Frequency Plasma Polymerization (RF-Plasma) of $\varepsilon$-Caprolactone and DEGDME for generation of PCL-PEG copolymer on the membrane (cellophane). B. Schematic of the multi-stack PCL-PEG coating for a tuneable release of entrapped drug layer (cis-platinum). 
contained the drug, Glisson's capsule was intact. In contrast, when implants contained the drug, the release induced the rupture of the Glisson's capsule and consequently, the liver parenchyma was destroyed. The presence of apoptotic cells only in liver parenchyma in the presence of the implant which encapsulated the drug suggested the efficacy of nano thick multilayer drug delivery membrane. These implants provided the release of high amounts of the drug without any complication in surrounding organs stomach, spleen, diaphragm, and others digestive organs including renal toxicity (Supplementary Results, Figure S-1). All the 20 mice were alive and healthy on Day 10.

In the present work, the histological examination of the liver tissues which were in contact with cisplatinum loaded plasma polymerized cellophane implants were showed hepatocellular vacuolization and cis-platinum induced inflammation of cells. As compared to untreated cellophane implanted on liver tissues, the apoptotic (TUNEL positive) cells were remained in the liver tissues which were in contact with cis-platinum loaded implants. Herein, we have shown the advantage of the one step, dry, clean, catalyst and solvent free soft plasma polymerized drug delivery system which can be prepared on a wide range of substrates and to achieve high purity materials and interfaces for in vitro and in vivo biological and clinical applications. In previous works, we used a wet chemical targeting method for ganciclovir-loaded albumin nanoparticles for intra ocular injection [28]. Co polymerization of the materials destined for nanoparticles generation, needed catalyser and solvent. Here, compared with traditional methods, we proposed plasma process which allows copolymerizing by means of a catalyst and solvent free process to regulate the drug release from a substrate.

However, we need further investigation to demonstrate the exact mechanism for loading and controlled release of cis-platinum induced hepatotoxicity and dose dependent effects on cancer tissues. In conclusion, low pressure plasma coating technique may be used to deposit coatings of uniform thicknesses over a variety of substrates for biological and clinical applications.

\section{MATERIALS AND METHODS}

\section{Animals}

A total of 20 female BALB/c mice (4 weeks old) were purchased from Charles River Laboratories (F 69592 L'Arbresle) France and body weight ranging from 20 to 30 g. All animals were maintained at the animal center for 2 weeks of adaptive feeding prior the start of experiment. Animals were randomly divided into three experimental groups and a control group. The mice were caged in groups of five in an air-filtered laminar flow cabinet and fed with irradiated food and autoclaved reverse-osmosis treated water. All procedures were performed under sterile conditions in a laminar flow hood. Five animals for each group were used. The experimental protocol was approved by the Ethics Review Committee for Animal Experimentation of UPMC, France. All experimental protocols were performed in accordance with the European Convention for the protection of vertebrate animals used for experimental and other scientific purposes (Council of Europe, 1986, ETS No. 123).

\section{Materials}

ع-CL (Purity: 97\%, MW: 114, Empirical formula: $\mathrm{C}_{6} \mathrm{H}_{10} \mathrm{O}_{2}$ ) and DEGME (Purity: 99.5\%, MW: 134.17, linear formula: $\left.\left(\mathrm{CH}_{3} \mathrm{OCH}_{2} \mathrm{CH}_{2}\right)_{2} \mathrm{O}\right)$ were purchased from Sigma Aldrich, France and used in this study without further purifications. For the drug delivery implant applications, a $50 \mu \mathrm{m}$ thick sheet of Cellulose bio-polymer was purchased from Humeau laboratories France. Cellophane substrates were cut to sizes of approximately $2 \mathrm{~cm}^{2}$ for all experiments.

\section{Antineoplastic drug}

Cisplatin or cis-diamminedichloroplatinum (II) (CDDP) (linear formula: $\mathrm{PtCl}_{2} \mathrm{H}_{6} \mathrm{~N}_{2}, \mathrm{MW}$ : 300.05) was used as an antineoplastic (anticancer) drug in sterile aqueous solution. Each $\mathrm{mL}$ of cisplatin solution, was used for the current study contained $1 \mathrm{mg}$ of cisplatin and 9 $\mathrm{mg}$ sodium chloride in water $(\mathrm{pH} 3.5-4.5)$. For the in vivo drug release study, $20 \mu \mathrm{l}$ of cisplatin solution of $1 \mathrm{mM}$ concentration in $1 \mathrm{X}$ PBS was dropped onto cellophane substrates pre-coated with plasma polymerized PEG (pDEGME) and allowed to dry at room temperature. The cellophane slides $(2 \mathrm{~cm} 2)$ loaded with cisplatin $(118 \mu \mathrm{gm} /$ $\mathrm{cm} 2)$ were immersed in $1 \mathrm{~mL}$ of $1 \mathrm{X}$ PBS (pH 7.0) at $37^{\circ} \mathrm{C}$. The sample was agitated for 5 minute with a vortex mixer, then sonicated (Ultrasonic Cleaner, Fisher Scientific, France) in a room temperature water bath for 5 minute, and mixed with a vortex mixer for an additional minute. Afterwards, the supernatant was collected and the cisplatin loading was determined by UV Visible spectroscopic (SAFAS UVmc2, France) measurements. Spectral data was collected from 180-500 nm, (Supplementary Results, Figure S-2).

\section{Plasma polymerization of PCL-co-PEG coatings}

PCL and PEG (PCL-co-PEG) copolymer coatings were fabricated in a low pressure inductively excited radio frequency-tubular quartz plasma reactor system ( $5 \mathrm{~cm}$ diameter, $40 \mathrm{~cm}$ length, base pressure of $3 \times 10^{-2}$ mbar). The schematics of plasma deposition setup for PCL-PEG coatings, technical details of the process and in vitro characterization of cis-platinum release as well 
as the cell-surface interactions have been provided in our earlier work [26]. Briefly, prior to each experimental run, the reactor was scrubbed and cleaned with detergent, organic solvents and dried using compressed air. After cleaning with solvent, the plasma reactor was reassembled and cleaned further with $30 \mathrm{~W}$ argon plasma discharge at $0.5 \mathrm{mbar}$ pressure for $30 \mathrm{~min}$. The partial pressure ratio of the two monomer fed in the reactor was controlled by the flow rate of carrier gas (i.e. Ar), which was regulated and measured by electronic mass flow controllers (MKS instruments). The flow rate of carrier gas was varied from 5 to $20 \mathrm{sccm}$ with the increment of $5 \mathrm{sccm}$ which was denoted by " 1 " for $5 \mathrm{sccm}$ and " 4 " for $20 \mathrm{sccm}$ respectively. The total flow rate was varied from $5 \mathrm{sccm}$ to $25 \mathrm{sccm}$ by keeping the operating pressure constant. The power was generated by Dressler Cesar RF generator which was delivered through the L-C matching network. The substrate is placed $9.0 \mathrm{~cm}$ below the coil. In the present work, we have deposited copolymer coatings for barrier layer at 20W CW (Continuous Wave) plasma which was then followed by deposition of a copolymer in a 1W PW (Pulse Wave). For the pulsed plasma discharge, the peak power $\left(\mathrm{P}_{\mathrm{pk}}\right)$ was $25 \mathrm{~W}$ and the duty cycle $(\mathrm{DC}=$ $\left(\mathrm{t}_{\text {on }} /\left(\mathrm{t}_{\text {on }}+\mathrm{t}_{\text {off }}\right)\right.$, where $\mathrm{t}_{\text {on }}$ and $\mathrm{t}_{\text {off }}$ were the 'plasma ON' and 'plasma OFF' times respectively) was $4 \%$ to obtain the effective plasma power $\left(\mathrm{P}_{\text {eff }}\right)$ which was $1 \mathrm{~W} \mathrm{PW}\left(\mathrm{t}_{\mathrm{on}}=\right.$ $4 \mathrm{~ms}$ and $\left.\mathrm{t}_{\text {off }}=96 \mathrm{~ms}\right)$. Plasma polymerization of organic monomers was carried out on biocompatible cellophane substrates. All the coatings were deposited for different time duration $\left(t_{d}\right)$. After polymer deposition, the reactor was again evacuated to base pressure before the plasma polymerization system was vented to atmospheric pressure with air.

\section{Determination of cis-platinum loading}

The cis-platinum solution was dispersed on pDEGME deposited cellophane substrates. In order to ensure cisplatinum recovery occurred from the cellophane implants, one implant was then cut into four individual pieces and submerged in $1 \mathrm{~mL}$ of $1 \mathrm{X}$ PBS (pH 7.0). The sample was agitated for 5 minute with a vortex mixer, then sonicated (Ultrasonic Cleaner, Fisher Scientific) in a room temperature water bath for 5 minute, and mixed with a vortex mixer for an additional minute. Afterwards, the supernatant was collected and the cis-platinum loading was determined by UV Visible spectroscopic (SAFAS UVmc ${ }^{2}$, France) measurements. Spectral data was collected from 180-500 nm.

\section{Scanning electron microscopy}

$10^{5} / 10 \mu \mathrm{l}$ of Suspended ovarian cancer cell line OVCAR-3 were deposited on a plasma polymerized glass and incubated for one minute then $2 \mathrm{ml}$ of RPMI medium (with $10 \%$ of Fetal Bovine Serum FBS, 1\% of L-Glutamine and $1 \%$ of Streptomycin) were added to the well. All the samples were incubated at $37^{\circ} \mathrm{C}$ and
$5 \% \mathrm{CO}_{2}$ for $48 \mathrm{~h}$. The culture medium was discarded and samples were washed. The samples were fixed in $2 \%$ glutaraldehyde and $1 \mathrm{X}$ phosphate buffer saline (PBS, $\mathrm{pH}$ 7.4) at room temperature for 1 hour, washed in $1 \mathrm{X}$ PBS and then post fixed in 1\% osmium-1X PBS for 45 min at room temperature and in dark conditions. After a final wash in $1 \mathrm{X}$ PBS, the samples were dehydrated in increasing concentrations of ethanol. Samples were dried by the critical point method with liquid $\mathrm{CO} 2$ and then sputter-coated with gold. They were observed with a S260 CAMBRIDGE scanning electron equipped with a $\mathrm{LaB}_{6}$ filament operating at $15 \mathrm{kV}$ and images were captured with the software "Orion" from $(\mathrm{NCH}$ Software).

\section{Preparation of implants}

A schematic of plasma deposited multilayer PCLco-PEG coatings on cis-platinum loaded cellophane for hepatic implantation is illustrated in Figure 6. For in vivo release of cis-platinum, the multilayer of plasma polymerized PCL-PEG copolymers were prepared on cellophane substrates as a multistep process discussed as follows: (i) $0^{\text {th }}$ layer: a hydrophilic layer of plasma polymerized DEGME (pDEGME) coating was deposited on $2 \mathrm{~cm}^{2}$ cellophane substrates for $5 \mathrm{~min}$ at $1 \mathrm{~W}$ effective power. (ii) Cis-platinum $\left(118 \mu \mathrm{gm} / \mathrm{cm}^{2}\right)$ solution was mobilized on pDEGME coated cellophane substrates. (iii) $1^{\text {st }}$ layer: To avoid the direct exposure of the RF plasma, PCL-co-PEG (1:4) coatings were deposited on cis-platinum loaded cellophane substrates under mild conditions $\left(\mathrm{P}_{\mathrm{pk}}=25 \mathrm{~W}, \mathrm{DC}=4 \%, \mathrm{P}_{\text {eff }}=1 \mathrm{~W}, \mathrm{t}_{\mathrm{d}}=5 \mathrm{~min}\right)$. (iv) $2^{\text {nd }}$ layer: The barrier layer coatings were deposited to encapsulate the drug under $20 \mathrm{~W} \mathrm{CW}$ power for 10 min. (v) $3^{\text {rd }}$ layer: Finally, PCL-co-PEG (1:4) coatings were deposited on top of the barrier layer under mild conditions for controlled cell-surface interactions (Figure 6). All the plasma deposited implants were prepared 1 hr before the in vivo experiments. All the experimental conditions for preparation of implants are summarized in Table 1.

\section{Membrane implantation}

All 20 animals were anesthetized with $2 \%$ isoflurane in oxygen with mechanical ventilation for 15 min. Each animal was fixed in a supine position. After skin disinfection with Betadine, a xifo-umbilical laparotomy was performed. A verification of the peritoneum and the abdominal cavity was realized and a self-retaining retractor was placed. If necessary, an Allis clamp type was placed on the xiphoid cartilage to improve exposure. The implants of cellophane $\left(2 \mathrm{~cm}^{2}\right)$ were placed above the left hepatic lobe, between the liver and the anterior face of the stomach. Untreated (Cello), plasma polymers ( $p P C L-c o-P E G-C e l l o)$ and plasma polymers + Cis-platinum (Cisp-pPCL-co-PEG- 
Table 1: Experimental conditions for preparation of drug delivery vehicles for in vivo implants

\begin{tabular}{|c|c|c|c|c|c|c|c|c|}
\hline Implant & Description & Cellophane & $\begin{array}{c}\text { pDEGME } \\
\left(0^{\text {th }} \text { layer }\right)\end{array}$ & $\begin{array}{c}\text { Cisplatin } \\
\text { loading }\end{array}$ & $\begin{array}{c}\text { Protective } \\
\text { coating } \\
\left(1^{\text {st layer })}\right.\end{array}$ & $\begin{array}{c}\text { Barrier } \\
\text { coating } \\
\left(2^{2 d} \text { layer }\right)\end{array}$ & $\begin{array}{c}\text { Functionalized } \\
\text { coating } \\
\left(3^{\text {rd }} \text { layer }\right)\end{array}$ & $\begin{array}{c}\text { No of } \\
\text { mice } \\
\text { per } \\
\text { group }\end{array}$ \\
\hline Control & & $X$ & $X$ & $X$ & $X$ & $X$ & $X$ & 5 \\
\hline Cello & $\begin{array}{l}\text { Untreated } \\
\text { Cellophane }\end{array}$ & $\sqrt{ }$ & $X$ & $X$ & $X$ & $X$ & $X$ & 5 \\
\hline $\begin{array}{l}\text { pPCL- } \\
\text { co-PEG- } \\
\text { Cello }\end{array}$ & $\begin{array}{l}\text { Cellophane } \\
\text { +Plasma } \\
\text { Polymers }\end{array}$ & $\sqrt{ }$ & $\sqrt{ }$ & $X$ & $\sqrt{ }$ & $\sqrt{ }$ & $\sqrt{ }$ & 5 \\
\hline $\begin{array}{l}\text { Cisp- } \\
\text { pPCL- } \\
\text { co-PEG- } \\
\text { Cello }\end{array}$ & $\begin{array}{l}\text { Cellophane } \\
\text { +Cisplatin } \\
\text { +Plasma } \\
\text { Polymers }\end{array}$ & $\sqrt{ }$ & $\sqrt{ }$ & $\sqrt{ }$ & $\sqrt{ }$ & $\sqrt{ }$ & $\sqrt{ }$ & 5 \\
\hline
\end{tabular}

Cello) treated cellophane were implanted into animals in the three different experimental groups (Table 1). In the control group, all animals were remained as received. The absorbable compress Surgicel ${ }^{\circledR}$ (Oxidized regenerated cellulose) was used to completely cover the implant and avoid the displacement (migration). The peritoneum was closed with a continuous suture of Prolene 7/0 (Ethicon, Somerville, NJ) a non-absorbable over lock or metal clips was placed on the skin. After surgical procedure, the anesthetics were discontinued, and all animals were allowed to recover for 20 minutes in a box flushed with $100 \%$ oxygen and then placed in their home cages. After 10 days from implantation, all mice from both experimental and control groups were euthanized by $\mathrm{CO}_{2}$ inhalation. General conditions of the surrounding tissue and the implanted cellophane substrates were observed with the naked eye. The part of liver opposite to implant was removed, fixed in PAF (4\%) and embedded in paraffin. The slides (4 micron) were produced and colored by hematein-eosin-safran (HES) according to classical methods in the anatomopathogical laboratory. Histopathological study of liver tissues was performed

\section{Terminal deoxynucleotidyl transferase dUTP nick-end labeling (TUNEL) assay}

After 10 days of implantation, the apoptotic cells in the liver tissues adjacent to the cellophane implants were detected using TUNEL (Promega, Madison, WI) assay. In brief, the sections of liver tissues were deparaffinined and dehydrated, followed by permeabilization with $20 \mu \mathrm{g} / \mathrm{mL}$ proteinase $\mathrm{K}$ and $0.2 \%$ Triton X-100 in 1X PBS. The slides were then labeled with a TdT reaction mixture for $90 \mathrm{~min}$ and were mounted with a mounting solution containing 4', 6-diamidino-2-phenylindole (DAPI). The apoptotic cells (green) and cell nucleus (blue) was examined using a fluorescence microscopy.

\section{In vivo release of cis-platinum}

The blood samples were collected from the mice retro orbital sinus into Heparin tubes after 10 days of implantation. The sampled blood $(0.3-0.5 \mathrm{~mL})$ were centrifuged at 3000 rpm for $10 \mathrm{~min}$ at $4^{\circ} \mathrm{C}$ to separate the plasma. Cis-platinum traces in the blood plasma was investigated using UV Vis Spectroscopic (SAFAS UV mc ${ }^{2}$, France) analysis. Spectral data was collected from 170-500 nm.

\section{ACKNOWLEDGMENTS}

We gratefully acknowledge University Pierre \& Marie Curie (UPMC), France for offering the PhD financial support (SB). We acknowledge Francoise Pillier. (SEM Platform, UPMC, Jussieu, Paris, France) for her help in scanning electron microscopy analysis and Jean Philippe Brouland for his help in anatomo-pathology studies.

\section{CONFLICTS OF INTEREST}

The authors declare no conflicts of interest.

\section{GRANT SUPPORT}

This work was supported by the France national cancer institute (INCA-2014-INSERM, CORAPLAS, a Research proposal in physics, mathematics and engineering sciences applied to the cancer research).

\section{Author contributions}

SB: designed and conducted plasma deposition experiments; analysed data; prepared figures; wrote the manuscript

FV: conducted in vivo experiments

JP: conducted plasma deposition experiments 
$\mathrm{RD}$ : conducted in vivo experiments

AB: plasma deposition experiments for SEM analysis

IA: pharmacology, cell biology and SEM analysis

MP: in vivo experiments (Director of INSER U965)

FAK: designed and conducted plasma deposition experiments; analysed data; prepared figures; wrote the manuscript (Director of LISE, Paris VI)

MM: designed and conducted in vivo experiments, analysed data, prepared figures, wrote the manuscript

\section{REFERENCES}

1. Kelland L. The resurgence of platinum-based cancer chemotherapy. Nature Reviews Cancer. 2007; 7: 573-584.

2. Rosenberg B, VanCamp L, Trosko JE, Mansour VH. Platinum compounds: A new class of potent antitumour agents. Nature. 1969; 222:385-386.

3. Jamieson ER, Lippard SJ. Structure, recognition, and processing of cis-platinum-DNA adducts. Chemical Reviews. 1999; 99:2467-2498.

4. Eastman A. Activation of programmed cell death by anticancer agents: cis-platinum as a model system. Cancer Cells. 1990; 2:275-280.

5. Sastry J, Kellie SJ. Severe neurotoxicity, ototoxicity and nephrotoxicity following high-dose cis-platinum and amifostine. Pediatric Hematology and Oncology. 2005; 22:441-445.

6. Sternberg CN, Donat SM, Bellmunt J, Millikan RE, Stadler W, De Mulder, P, Sherif A, von der Maase H, Tsukamoto T, Soloway, M. S. Chemotherapy for Bladder Cancer: Treatment Guidelines for Neoadjuvant Chemotherapy, Bladder Preservation, Adjuvant Chemotherapy, and Metastatic Cancer. Urology. 2007;69:62-79.

7. Ivanov AI, Christodoulou J, Parkinson JA, Barnham KJ, Tucker A, Woodrow J, Sadler PJ. Cis-platinum Binding Sites on Human Albumin. The Journal of Biological Chemistry. 1998;273: 14721-14730.

8. Wang D, Lippard SJ. Cellular processing of platinum anticancer drugs. Nature Reviews Drug Discovery. 2005; 4:307-320.

9. Cuong NV, Jiang JL, Li YL, Chen JR, Jwo SC, Hsieh MF. Doxorubicin-loaded PEG-PCL-PEG micelle using xenograft model of nude mice: Effect of multiple administration of micelle on the suppression of human breast cancer. Cancers. 2011; 3:61-78.

10. Dhar S, Kolishetti N, Lippard SJ, Farokhzad OC. Targeted delivery of a cis-platinum prodrug for safer and more effective prostate cancer therapy in vivo. Proceedings of the National Academy of Sciences. 2011; 108:1850-1855.

11. Krieger ML, Eckstein N, Schneider V, Koch M, Royer HD, Jaehde U, Bendas G. Overcoming cis-platinum resistance of ovarian cancer cells by targeted liposomes in vitro. The International Journal of Pharmaceutics. 2010; 389:10-17.

12. Nishiyama N, Yokoyama M, Aoyagi T, Okano T, Sakurai Y, Kataoka K. Preparation and characterization of self-assembled polymer-metal complex micelle from cisdichlorodiammineplatinum (II) and poly (ethylene glycol)poly ( $\alpha, \beta$-aspartic acid) block copolymer in an aqueous medium. Langmuir. 1999; 15:377-383.

13. Ye H, Jin L, Hu R, Yi Z, Li J, Wu Y, Xi X, Wu Z. Poly $(\gamma$, l-glutamic acid)-cis-platinum conjugate effectively inhibits human breast tumor xenografted in nude mice. Biomaterials. 2006; 27:5958-5965.

14. Aryal S, Jack Hu CM, Zhang L. Polymer-Cis-platinum Conjugate Nanoparticles for Acid-Responsive Drug Delivery. ACS Nano. 2010; 4:251-258.

15. Duan X, He C, Kron SJ, Lin W. Nanoparticle formulations of cis-platinum for cancer therapy. Wiley Interdisciplinary Reviews: Nanomedicine and Nanobiotechnology. 2016; doi: 10.1002/wnan.1390.

16. Zhao F, Zhao Y, Liu Y, Chang X, Chen C, Zhao Y. Cellular Uptake, Intracellular Trafficking, and Cytotoxicity of Nanomaterials. Small. 2011; 7:1322-1337.

17. Bhatt S, Pulpytel J, Ceccone G, Lisboa P, Rossi F, Kumar V, Arefi-Khonsari F. Nanostructured Protein Repellant Amphiphilic Copolymer Coatings with Optimized Surface Energy by Inductively Excited Low Pressure Plasma. Langmuir. 2011; 27:14570-14580.

18. Bhatt S, Pulpytel J, Arefi-Khonsari F. Low and Atmospheric Plasma Polymerization of Nanocoatings for Bio Applications. Surface Innovations. 2015; 3:63-83.

19. Khelifa F, Ershov S, Habibi Y, Snyders R, Dubois P. FreeRadical-Induced Grafting from Plasma Polymer Surfaces, Chemical Reviews. 2016; 116:3975-4005.

20. Bhatt S, Pulpytel J, Mirshahi M, Arefi-Khonsari F. Catalyst-Free Plasma-Assisted Copolymerization of Poly( $\varepsilon$-caprolactone)-poly(ethylene glycol) for Biomedical Applications. ACS Macro Letters. 2012; 1: 764-767.

21. Bhatt S, Pulpytel J, Mirshahi M, Arefi-Khonsari F. Nano thick poly ( $\varepsilon$-caprolactone)-poly (ethylene glycol) coatings developed by catalyst free plasma assisted copolymerization process for biomedical applications. RSC Advances. 2012; 2:9114-9123.

22. Bhatt S, Pulpytel J, Mirshahi M, Arefi-Khonsari F. Plasma Co-polymerized Nano Coatings - As a Biodegradable Solid Carrier for Controlled Drug Delivery Applications. Polymer. 2013; 54: 4820-4829.

23. Canal C, Khurana K, Gallinetti S, Bhatt S, Pulpytel J, ArefiKhonsari F, Ginebra MP. Design of calcium phosphate scaffolds with controlled simvastatin release by plasma polymerization. Polymer. 2016; 92:170-178.

24. Housecroft CE, Sharpe AG. Inorganic Chemistry, 2nd ed., Pearson Education Limited, Essex, England 2004.

25. Koc A, Duru M, Ciralik H, Akcan R, Sogut S. Protective agent, erdosteine, against cis-platinum-induced hepatic oxidant injury in rats. Molecular and Cellular Biochemistry. 2005; 278:79-84.

26. Trynda-Lemiesz L, Kozłowski H, Keppler BK. Effect of cis-, trans-diamminedichloroplatinum(II) and DBP on 
human serum albumin. Journal of Inorganic Biochemistry. 1999; 77:141-146.

27. Anilanmert B, Yalçin G, Ariöz F, Dölen E. The spectrophotometric determination of cisplatin in urine, using o-phenylenediamine as derivatizing agent. Analytical Letters. 2001; 34: 113-123.
28. Merodio M, Irache JM, Valamanesh F, Mirshahi M. Ocular disposition and tolerance of ganciclovir-loaded albumin nanoparticles after intravitreal injection in rats. Biomaterials. 2002; 23:1587-94. 